\title{
Does it Make Sense to Speak of Self-Locating Uncertainty in the Universal Wave Function? Remarks on Sebens and Carroll
}

\author{
Adrian Kent \\ Centre for Quantum Information and Foundations, DAMTP, \\ Centre for Mathematical Sciences, University of Cambridge, \\ Wilberforce Road, Cambridge, CB3 OWA, U.K. and \\ Perimeter Institute for Theoretical Physics, 31 Caroline Street North, Waterloo, ON N2L 2Y5, Canada.*
}

(Dated: August 2014)

\begin{abstract}
Following a proposal of Vaidman [19 21], Sebens and Carroll [4, 18] have argued that in Everettian (i.e. purely unitary) quantum theory, observers are uncertain, before they complete their observation, about which Everettian branch they are on. They argue further that this solves the problem of making sense of probabilities within Everettian quantum theory, even though the theory itself is deterministic. We note some problems with these arguments.
\end{abstract}

\section{INTRODUCTION}

Everett's idea 7] that pure unitary quantum evolution holds at all times continues to fascinate many theoretical physicists.

Making scientific sense of Everett's idea is difficult, as evidenced by the many and generally incompatible attempts (see e.g. [5, [, , 8 - 10, 17, 21, 24] and references therein) to show how unitary quantum theory explains the appearance of a quasiclassical world and the apparent validity of the Born rule and Copenhagen quantum theory, and evidenced also by the problems with all of these attempts (see e.g. [1 $[3,12,13,15]$ ). There is still nothing close to a consensus on the most promising way forward, even among many-worlds enthusiasts. This adds motivation for developing alternative ways of formulating quantum theory (e.g. 14]) that have the purported advantages of many-worlds ideas - realism, and Lorentz invariance - but describe a single real world, so avoiding both the conceptual problems and the fantastic nature of many-worlds ideas. Still, for many, the appeal of many-worlds ideas evidently persists.

This note looks at recent suggestions by Sebens and Carroll [4, 18], who take up a proposal originally made by Vaidman [19 21]. Sebens and Carroll "seek to provide an epistemic - as opposed to a decision-theoretic - derivation of the Born rule which connects quantum uncertainty to the sort of self-locating uncertainty present in very large universes" ([18], p. 3). Our aim here is not to give a full critique of their work, nor to address Vaidman's earlier papers directly, but simply to note some problems with Sebens and Carroll's general approach. These seem to us likely sufficient to doom their attempt to make sense of many-worlds probabilities via self-locating uncertainty. That said, we do not give a logical proof that no ideas along these lines can ever work. Our discussion should, we hope, in any case, help readers to focus on unresolved issues and form their own views about whether or how they could be resolved.

We focus on the question of whether it is meaningful to say that observers are uncertain about their self-location in the universal wave function, and can meaningfully assign probabilities to the events that they are located in one branch or another. We thus do not address Sebens and Carroll's purported derivation of the Born rule, except in the sense that this derivation obviously fails if there are no relevant probabilities to discuss [25].

As Sebens and Carroll note, one of the key difficulties for Everettians is finding any well-defined role for the numbers that, in standard quantum theory, are defined by the Born rule to be probabilities. This is a problem since unitary quantum theory is deterministic, and so on the face of it there is nothing to be uncertain about. Yet, of course, standard quantum theory is universally seen as successful precisely because it makes quantitative probabilistic predictions that have been confirmed (or, more precisely, not statistically falsified) by all experiments to date. If quantum theory simply said that, when we carry out a quantum experiment, in some unobservable sense every possible outcome happens, and that we might observe any one of these possible outcomes, no one would ever have taken it seriously as a scientific theory.

Sebens and Carroll [4] introduce their key idea within a standard toy model of an ideal measurement of a spin $1 / 2$ particle, involving an apparatus $A$, an observer $O$ and the environment $\omega$, whose pre-measurement states are $1 / \sqrt{2}(|\uparrow\rangle+|\downarrow\rangle),\left|A_{0}\right\rangle,\left|O_{0}\right\rangle,\left|\omega_{0}\right\rangle$ respectively. The measurement is modelled by successive entangling unitaries, so that, neglecting normalization, we have

$$
\begin{aligned}
\left|O_{0}\right\rangle(|\uparrow\rangle+|\downarrow\rangle)\left|A_{0}\right\rangle\left|\omega_{0}\right\rangle & \rightarrow\left|O_{0}\right\rangle\left(|\uparrow\rangle\left|A_{\uparrow}\right\rangle+|\downarrow\rangle\left|A_{\downarrow}\right\rangle\right)\left|\omega_{0}\right\rangle \\
& \rightarrow\left|O_{0}\right\rangle\left(|\uparrow\rangle\left|A_{\uparrow}\right\rangle\left|\omega_{\uparrow}\right\rangle+|\downarrow\rangle\left|A_{\downarrow}\right\rangle\left|\omega_{\downarrow}\right\rangle\right)
\end{aligned}
$$




$$
\begin{aligned}
& =\left|O_{0}\right\rangle|\uparrow\rangle\left|A_{\uparrow}\right\rangle\left|\omega_{\uparrow}\right\rangle+\left|O_{0}\right\rangle|\downarrow\rangle\left|A_{\downarrow}\right\rangle\left|\omega_{\downarrow}\right\rangle \\
& \rightarrow\left|O_{\uparrow}\right\rangle|\uparrow\rangle\left|A_{\uparrow}\right\rangle\left|\omega_{\uparrow}\right\rangle+\left|O_{\downarrow}\right\rangle|\downarrow\rangle\left|A_{\downarrow}\right\rangle\left|\omega_{\downarrow}\right\rangle .
\end{aligned}
$$

Here we follow [4] in highlighting the identity between (2) and (3), for clarity.

Roughly speaking (in the sort of language that physicists ordinarily use when they are talking about quantum experiments and not worrying about conceptual rigour), then, in this model, first the particle interacts with the apparatus, which then interacts with the environment, and finally - via direct inspection - with the observer. The resulting states $\left|X_{\uparrow}\right\rangle$ and $\left|X_{\downarrow}\right\rangle$ are taken to be orthogonal, for $X=O, A$ or $\omega$.

Of course, to examine what role, if any, probability might have in Everettian quantum theory one needs to be much more careful than this in explaining how physical events might be associated with the mathematics. So we turn now to the words Sebens and Carroll attach to these equations. First, they state that at the second (and third) line, "the wave function has branched - decoherence has occurred, as indicated by the different environment states".

There is more that should be explained here. Is this notion of branching inherently fuzzy? Does it require the relevant environment states to be precisely orthogonal or only approximately? Can we speak of branchings for any decomposition of the Hilbert space into tensor products, or is it only defined for observers and environments, and if the latter, what precisely (or at least approximately) is an observer and an environment?

Still, one could read the quoted statement, taken in isolation, as roughly consistent with other modern Everettian ideas (e.g. 22, 23]), in which branches are not fundamental, nor precisely defined, but concepts we impose on the universal wave function for our own convenience, using approximate definitions of observer and environment. On this view, in more realistic models, neither the total number of branches, nor the number of branches associated with any given component of the wave function, is well defined. (Sebens and Carroll explicitly agree with this last point: see for example footnote 10 of [18].) Measurement-like interactions are happening throughout space-time, and it's a largely arbitrary choice which and how many of them one chooses to represent explicitly in a decomposition of the wave function. So long as the components identified are at least approximately orthogonal and remain so for at least a short while, we are free to call them branches if we wish.

So far, then, arguably, perhaps, so good. "The wave function has branched." could be read as just shorthand for something like "Let's write the wave function in the form (3), which gives a nice (although not objectively significant) way of thinking about the mathematics."

But now Sebens and Carroll add "The observer is still described by a unique state $\left|O_{0}\right\rangle$, but there are two copies, one in each branch. Such an observer (who by construction doesn't yet know the result of the measurement) is in a state of self-locating uncertainty." These are bold, and prima facie peculiar, assertions, which seem to need careful justification and explanation.

To elaborate:

There are, of course, two copies of the expression $\left|O_{0}\right\rangle$ written in equation (3) above. The equivalent expression (2) uses just one copy. But, of course, so far these are just statements about ink on paper. To translate them into statements about one or more observers, who are uncertain about some relevant fact about their location on branches, requires some principled general account of how we start from the universal wave function and derive an ontology that includes (at least) observers and branches. Moreover, Sebens and Carroll need this account to give particular answers: observers must be split into copies before they observe the relevant event; these copies must at that point in time be located on definite branches; each must, however, be uncertain about the identity of their own branch.

To see that this is likely to be a difficult project, one need only list some obvious questions.

Firstly, (why) can we be sure that the post-measurement/pre-observation state in this toy model does represent two copies of an observer? (Why) can we not say, for example, looking at equation (2), that it represents one observer? On this latter view, our sole observer has not interacted with the entangled particle-apparatus-environment state, and has no uncertainty about this state, or their "branch location", or anything else. This seems perfectly coherent, and indeed much simpler and more natural than the picture Sebens and Carroll suggest. Is it somehow logically flawed? If not, what justifies the confident assertion that it is wrong, and the two-copy picture is right?

Secondly, what actually is the status of branches meant to be? Sebens and Carroll's self-locating uncertainty seems to be meant to be an uncertainty about an objective fact about reality: either I am the observer on the up branch or the observer on the down branch, but I'm not sure which. But then how can these be possible facts about reality, when the choice of branches is agreed to be not objective, but rather an arbitrary mathematical choice which - remember can be made in many generally incompatible ways in generic models? It seems as though, in a more detailed model, we would need to say that there is no fixed number of copies of observers or branches: how exactly, then, are we supposed to speak of the self-location problem of an observer, and what is it that they are supposed to be uncertain about? 
Thirdly, suppose for the sake of the argument that (contra Wallace [22, 23] and Sebens-Carroll [4, 18] and most other modern Everettians) we were able and willing to somehow postulate some natural choice of branching structure as objective. Or suppose (which might be closer to Sebens and Carroll's intentions) that we have postulates that allow at least some facts about the branching structure to be declared objective. Even then, exactly how and why would this imply objective facts about observers splitting - before they make their observations - into many copies? Is this weird pre-observation splitting of observers supposed to happen the instant a measurement interaction is completed? If so, with respect to which reference frame? And are we happy to postulate a story about an underlying objective reality that thus not only breaks Lorentz symmetry but also proposes that measurement events superluminally cause observer splittings? If not, are we supposed to postulate a picture in which some mysterious observer-splitting influence propagates at light speed, radiating outwards from the location of measurement interactions? Or were all observers split from the moment of birth, before many of the measurements they later observe took place, with each copy destined in advance to see a particular set of measurement outcomes?

And, in any of these cases, what about interactions that are reversed through recoherence? Or interactions that produce approximate but not complete decoherence?

Finally, but importantly, and related to the last questions, how exactly are Sebens and Carroll's self-uncertain observers supposed to evolve over time? Are their splits supposed to be instantaneous? If not, what happens during the splitting process? Do sequences of splits always simply subdivide pre-existing observers - so that we have a treelike branching in observer space - or can they sometimes (for instance, when unobserved external systems decohere and then recohere) recombine?

\section{REDUCED DENSITY MATRICES AND BRANCHING}

Sebens and Carroll present another version of their proposal in [18], framed in terms of reduced density matrices. Specifically, they suggest a principle they call ESP-QM (18], p.15):

"Suppose that an experiment has just measured observable $\hat{O}$ of system $S$ and registered some eigenvalue $O_{i}$ on each branch of the wave function. The probability that agent $A$ ought to assign to the detector $D$ having registered $O_{i}$ when the universal wave function is $\Psi, P\left(O_{i} \mid \Psi\right)$, only depends on the reduced density matrix of $A$ and $D, \hat{\rho}_{A D}$ :

$$
P\left(O_{i} \mid \Psi\right)=P\left(O_{i} \mid \hat{\rho}_{A D}\right) . "
$$

The wording of this version, Sebens and Carroll claim ([18], p.15), allows them "to shake the unrealistic assumption that the number of branches in which a certain outcome occurs is well-defined". As noted above, the assumption is indeed generally agreed to be unrealistic, so this would be good. Again, though, in this version, Sebens and Carroll attach their own narratives to equations without any principled justification. What is supposed to be meant by the event "the detector $D$ having registered $O_{i}$ " to which agent $A$ is supposed to assign a probability? It clearly isn't the event "the detector $D$ registered $O_{i}$ in at least one branch", which, given the understanding of branching Sebens and Carroll want to adopt, has probability one. Could it be the event "the detector $D$ registered $O_{i}$ on my branch"? It seems it must be: what other options are there? But then we return to the formulation of [4], and all the associated problems noted above.

\section{SUMMARY}

Sebens and Carroll's attempts [4, 18] to find a role for probabilities in many-worlds quantum theory via self-locating uncertainty are puzzling, since as presented they seem to rely on simply plucking assertions from thin air. Fifty-seven years of sometimes careful work (e.g. 22, 3, 5, 6, 8 10, 17]) on trying to make scientific sense of Everettian quantum theory ought, surely, to have persuaded the theoretical physics community that one cannot make useful progress this way. Wherever one thinks of the scientific status of many worlds quantum theory, one cannot reasonably, at this point, think it is so obvious how to translate equations into statements about a many-worlds reality that arguments and explanations are redundant.

Perhaps Sebens and Carroll are appealing to unarticulated beliefs about an underlying Everettian ontology - some careful account of how branches and observers can be identified from the universal wave function - that supports their assertions. Unless and until a clearer account is given, it is hard to know whether a consistent ontology of the form Sebens and Carroll require exists at all. 
The most familiar example of an Everettian ontology that could perhaps be adapted for Sebens and Carroll's purposes is Albert and Loewer's unloved "many-minds interpretation" 2], which essentially everyone, including Albert and Loewer, agrees is utterly unsatisfactory.

Another possible option available to try to justify Sebens and Carroll's assertions is to search for a selection criterion that either picks out one particular branching structure, or a class of approximately equivalent branching structures. The idea here would be that, given a theory of the initial state and a unitary evolution law, one can algorithmically define a branching structure using simple postulates. Proposals for postulates of this type have recently been made by Riedel et al. [16]. While intriguing, the ideas of [16] are at present tentative. In particular, the branching rules studied in [16] are inherently non-relativistic, and so give no hint of how to address the questions we listed concerning the consistency of Sebens and Carroll's ideas with special relativity (let alone general relativity or quantum gravity). The rules given in [16] are also defined only for a pure initial state.

Moreover, it is worth underlining again here that, if we were able to find reasonably natural postulates that respected physical symmetries and defined an objective branching structure for the universal wave function, it would be superfluous to postulate many independent real worlds. It would be simpler and more natural to postulate that precisely one of the branches is randomly chosen (using the Born weight distribution) and realised in nature.

In summary, the Everettian literature to date strongly suggests that it will be hard for Sebens and Carroll to find any ontology supporting their ideas. It seems to us that any ontology that might possibly do so would be so baroque and ad hoc that it would entirely undercut the case for the simplicity and elegance of Everettian quantum theory. Of course, those who continue to see self-locating uncertainty as a potentially useful notion in the context of many worlds quantum theory will take this as a challenge. We hope that our discussion has at least helped outline the scale of this challenge.

\section{ACKNOWLEDGEMENTS}

I am grateful to Charles Bennett, Sean Carroll, James Hartle, Ruth Kastner, Jess Riedel, Charles Sebens, Graeme Smith, John Smolin, Mark Srednicki, Lev Vaidman, Michael Zwolak and Wojciech Zurek for very helpful comments and discussions. I also thank Charles Bennett and Jess Riedel for organising the August 2014 workshop "Quantum Foundations of a Classical Universe", at which several of these discussions took place. This work was partially supported by a grants from the John Templeton Foundation and from FQXi, and by Perimeter Institute for Theoretical Physics. Research at Perimeter Institute is supported by the Government of Canada through Industry Canada and by the Province of Ontario through the Ministry of Research and Innovation.

\section{References}

* Electronic address: A.P.A.Kent@damtp.cam.ac.uk

[1] D. Albert. Probability in the Everett picture. In S. Saunders, J. Barrett, A. Kent, and D. Wallace, editors, Many Worlds?: Everett, Quantum Theory, and Reality, pages 355-368. Oxford University Press, 2010.

[2] D. Albert and B. Loewer. Interpreting the many worlds interpretation. Synthese, 77(2):195-213, 1988.

[3] J.S. Bell. Speakable and Unspeakable in Quantum Mechanics. Cambridge University Press, 2004.

[4] Sean M Carroll and Charles T Sebens. Many worlds, the Born rule, and self-locating uncertainty. In Quantum Theory: A Two-Time Success Story, pages 157-169. Springer Milan, 2014.

[5] D. Deutsch. Comment on Lockwood. The British Journal for the Philosophy of Science, 47(2):222-228, 1996.

[6] B.S. DeWitt and N. Graham. The Many-Worlds Interpretation of Quantum Mechanics. Princeton Series in Physics. Books on Demand.

[7] Hugh Everett III. "Relative state" formulation of quantum mechanics. Reviews of modern physics, $29(3): 454,1957$.

[8] M. Gell-Mann and J.B. Hartle. Quantum mechanics in the light of quantum cosmology. In W. Zurek, editor, Complexity, entropy and the physics of information, pages 425-458. Addison Wesley, Reading, 1990.

[9] R. Geroch. The Everett interpretation. Nô̂s, 18(4):617-633, 1984.

[10] J.B. Hartle. The quantum mechanics of cosmology. In T. Piran S.Coleman, J. Hartle and S. Weinberg, editors, Quantum Cosmology and Baby Universes: Proceedings of the 7th Jerusalem Winter School for Theoretical Physics, pages 65-157. World Scientific, 1991.

[11] Ruth E Kastner. Einselection of pointer observables: The new h-theorem? Studies in History and Philosophy of Science Part B: Studies in History and Philosophy of Modern Physics, 2014. 
[12] A. Kent. Against many-worlds interpretations. International Journal of Modern Physics, page 1745, 1990.

[13] A. Kent. One world versus many: The inadequacy of Everettian accounts of evolution, probability, and scientific confirmation. In S Saunders, J. Barrett, A. Kent, and D. Wallace, editors, Many worlds?: Everett, quantum theory, and reality, pages 307-354. Oxford University Press, 2010. arXiv:0905.0624.

[14] A. Kent. Solution to the Lorentzian quantum reality problem. Phys. Rev. A, 90:012107, Jul 2014.

[15] H. Price. Decisions, decisions, decisions: Can Everett salvage Savage? In S. Saunders, J. Barrett, A. Kent, and D. Wallace, editors, Many Worlds?: Everett, Quantum Theory, and Reality, pages 369-390. Oxford University Press, 2010.

[16] C. Jess Riedel, Wojciech H. Zurek, and Michael Zwolak. The Objective Past of a Quantum Universe - Part 1: Redundant Records of Consistent Histories. 2013.

[17] S. Saunders, J. Barrett, A. Kent, and D. Wallace. Many Worlds?: Everett, Quantum Theory, and Reality. Oxford University Press, 2010.

[18] Charles T Sebens and Sean M Carroll. Self-locating uncertainty and the origin of probability in Everettian quantum mechanics. arXiv preprint arXiv:1405.7577, 2014.

[19] L. Vaidman. Schizophrenic experiences of the neutron or why we should believe in the many-worlds interpretation of quantum theory. International Studies in the Philosophy of Science, 12:245-261, 1998.

[20] L. Vaidman. Many-worlds interpretation of quantum mechanics. In Edward N. Zalta, editor, The Stanford Encyclopedia of Philosophy, 2008.

[21] L. Vaidman. Probability in the many-worlds interpretation of quantum mechanics. In Y. Ben-Menahem and M. Hemmo, editors, The Probable and the Improbable: Understanding Probability in Physics, Essays in Memory of Itamar Pitowsky. Springer, 2011.

[22] D. Wallace. Decoherence and ontology. In S Saunders, J. Barrett, A. Kent, and D. Wallace, editors, Many worlds?: Everett, quantum theory, and reality, pages 53-72. Oxford University Press, 2010.

[23] David Wallace. The emergent multiverse: Quantum theory according to the Everett interpretation. Oxford University Press, 2012.

[24] W.H. Zurek. Quantum Darwinism. Nature Physics, 5(3):181-188, 2009.

[25] For a recent critique of various proposed derivations of the Born rule, see [11]. 